\title{
Existence and multiple solutions for a critical quasilinear equation with singular potentials
}

\author{
Shaowei Chen and Zhi-Qiang Wang
}

Abstract. We study the following quasilinear elliptic equations

$$
-\Delta_{p} u+V(x)|u|^{p-2} u=K(x)|u|^{q-2} u \text { in } \mathbb{R}^{N}
$$

where $1<p<N$ and $q=p(N-p s / b) /(N-p)$ with constants $b$ and $s$ such that $b<p, b \neq 0,0<\frac{s}{b}<1$. This exponent $q$ behaves like a critical exponent due to the presence of the potentials even though $p<$ $q<p^{*}=\frac{p N}{N-p}$ the Sobolev critical exponent. The potential functions $V$ and $K$ are locally bounded functions and satisfy that there exist positive constants $L, C_{1}, C_{2}, D_{1}$ and $D_{2}$ such that $C_{1} \leq|x|^{b} V(x) \leq C_{2}$ and $D_{1} \leq$ $|x|^{s} K(x) \leq D_{2}$ for $|x| \geq L$. We prove that below some energy threshold, the Palais-Smale condition holds for the functional corresponding to this equation. And we show that the finite energy solutions of this equation have exponential decay like $e^{-\gamma|x|^{1-b / p}}$ at infinity. If $V$ has a critical frequency, i.e., $V^{-1}(0)$ has a non-empty interior, we prove that

$$
-\Delta_{p} u+\lambda V(x)|u|^{p-2} u=K(x)|u|^{q-2} u \text { in } \mathbb{R}^{N}
$$

has more and more solutions as $\lambda \rightarrow+\infty$.

Mathematics Subject Classification. 35J20, 35J60.

Keywords. Quasilinear elliptic equation, Critical exponent, Decaying or coercive potentials, Exponential decay.

\section{Introduction and statement of results}

In this paper, we consider the following quasilinear elliptic equation

$$
-\Delta_{p} u+V(x)|u|^{p-2} u=K(x)|u|^{q-2} u \text { in } \mathbb{R}^{N}, u(x) \rightarrow 0 \text { as }|x| \rightarrow \infty
$$

where $N \geq 2,1<p<N$ and $\Delta_{p} u=\operatorname{div}\left(|\nabla u|^{p-2} \nabla u\right)$. The exponent

$$
q=\frac{p\left(N-\frac{p s}{b}\right)}{N-p}
$$


with the real numbers $b$ and $s$ satisfying

$$
b<p, \quad b \neq 0, \quad 0<\frac{s}{b}<1 .
$$

By this definition, it is easy to see $p<q<p^{*}:=p N /(N-p)$ with $p^{*}$ the Sobolev critical exponent. Even though $q<p^{*}$ we will see that $q$ behaves like a critical exponent due to the effects of the potential functions $V$ and $K$ which may have singular asymptotic near infinity.

For the potential functions $V$ and $K$, we assume

$\left(\mathbf{A}_{1}\right) . V$ and $K$ are locally bounded nonnegative functions in $\mathbb{R}^{N}$.

$\left(\mathbf{A}_{2}\right)$. There exist positive constants $L, C_{1}, C_{2}, D_{1}$ and $D_{2}$ such that, for $|x| \geq L$

$$
C_{1} \leq|x|^{b} V(x) \leq C_{2}, D_{1} \leq|x|^{s} K(x) \leq D_{2} .
$$

When $0<b<p, V$ and $K$ decay to zero at infinity and when $b<0, V$ and $K$ are coercive.

Equation (1.1) arises in various applications, such as superfluidity, plasticity, population genetics, chemical reactor theory, and the study of standing wave solutions of certain nonlinear Schrödinger equations. Therefore, it has received growing attention in recent years (we refer to [2$4,6,9,12,21,22,26,29,30]$ for more references therein).

There have been mathematical works for related problems in recent years. When $p=2, b>0$ and $2(N-2 s / b) /(N-2)<q<2^{*}$, Ambrosetti et al. [5] proved that (1.1) has a nonzero solution under the assumptions $\left(\mathbf{A}_{\mathbf{1}}\right)$ and $\left(\mathbf{A}_{\mathbf{2}}\right)$. In fact, it was proved in [5] that there is a compact embedding from a weighted Sobolev space into a weighted $L^{q}$ space, which provides the compactness of the variational formulation. However, the case $q=2(N-2 s / b) /(N-2)$ was left open in [5] since for $q=2(N-2 s / b) /(N-2)$ the compact embedding breaks down in general and in a sense it is a critical exponent problem as this $q$ appears on the boundary of the embedding range. For general $p \in(1, N)$, Lyberopoulos proved in [15] (see also [16]) that when $b>0,1<p<N$ and $p(N-p s / b) /(N-$ $p)<q<p^{*},(1.1)$ has a nonzero solution if $V$ and $K$ satisfy $\left(\mathbf{A}_{\mathbf{1}}\right)$ and $\left(\mathbf{A}_{\mathbf{2}}\right)$. When $V$ and $K$ are radially symmetric functions, existence of solutions for (1.1) can be obtained through some compact embedding theorems of weighted Sobolev spaces. Again the critical case $q=p(N-p s / b) /(N-p)$ was not treated in $[15,16]$, We also mention $[20,21,23-26]$ in which cases, certain compact embedding was proved so the Euler-Lagrange functional corresponding to (1.1) satisfies the Palais-Smale condition and the nonzero solution of (1.1) can be obtained through the standard critical point theorems.

When $b$ and $s$ satisfy (1.3), $q=p(N-p s / b) /(N-p)$ and $V$ and $K$ satisfy $\left(\mathbf{A}_{\mathbf{1}}\right)$ and $\left(\mathbf{A}_{\mathbf{2}}\right)$, the functional corresponding to (1.1) does not satisfy the Palais-Smale condition in general. From this point of view, $q=$ $p(N-p s / b) /(N-p)$ should be seen as a kind of critical exponent for Eq. (1.1). For the case $p=2$, in [11], through a transformation, Chen obtained an equivalent equation for (1.1) and then, using the concentration-compactness principle, he got a global compactness theorem for this equivalent equation. This theorem reveals why the Palais-Smale condition does not holds. Using this 
theorem, he obtained a nonzero solution for (1.1) under some additional conditions. However, the method in [11] fails for the general case $p \neq 2$, and it seems difficult to find a similar transform for (1.1) as the case $p=2$. In this paper, we develop a new approach, which unifies all cases $p>1$, for the existence of solutions of (1.1). We investigate the compactness first and find that below some energy threshold, the functional corresponding to (1.1) satisfies the Palais-Smale condition (Theorem 3.3). As a consequence, we provide a sufficient condition to insure the existence of a minimizer for the infimum (3.1) (Corollary 3.4), which corresponds to a nonzero solution of (1.1).

In the second part of the paper we study the following equation with a parameter $\lambda>0$

$$
-\Delta_{p} u+\lambda V(x)|u|^{p-2} u=K(x)|u|^{q-2} u \quad \text { in } \mathbb{R}^{N}, \quad|u(x)| \rightarrow 0 \quad \text { as }|x| \rightarrow \infty
$$

where $V$ has a critical frequency in the sense that the interior of the set $Z=$ $V^{-1}(0)$ is non-empty. This is a steep potential well problem with $\lambda$ controlling the depth of the potential. We prove that for any $m \in \mathbb{N}$, there exists $\Lambda_{m}>0$ such that when $\lambda \geq \Lambda_{m}$, this equation has at least $m$ solutions (Theorem 4.2) and for fixed $m \in \mathbb{N}$, as $\lambda \rightarrow \infty$, these solutions concentrate on $Z$ (Theorem 4.3). These results generalize the classical work of Beyon, Bartsch, Pankov and Wang for semilinear Schrödinger equations (see $[7,8,10,13])$ to the quasilinear Schrödinger equations. Finally, we prove that the finite energy solutions for (1.1) have $e^{-\gamma|x|^{1-b / p}}$ decay at infinity, where $\gamma>0$ (Theorem 5.1).

Notation. $B(a, r)$ denotes the open ball of radius $r$ and centered at $a$ in $\mathbb{R}^{N}$. For a Banach space $E$, we denote the dual space of $E$ by $E^{\prime}$, and denote the strong and the weak convergences in $E$ by $\rightarrow$ and $\rightarrow$, respectively. For $\varphi \in C^{1}(E ; \mathbb{R})$, we denote the Fréchet derivative of $\varphi$ at $u$ by $\varphi^{\prime}(u)$. The Gateaux derivative of $\varphi$ is denoted by $\left\langle\varphi^{\prime}(u), v\right\rangle, \forall u, v \in E . L_{l o c}^{\infty}\left(\mathbb{R}^{N}\right)$ denotes the space of locally bounded functions in $\mathbb{R}^{N}$. Let $\Omega$ be a domain in $\mathbb{R}^{N}$. $C_{0}^{\infty}(\Omega)$ denotes the space of smooth functions with compact support in $\Omega$.

\section{Variational structure for Eq. (1.1)}

In this section, we shall prove that under the assumptions $\left(\mathbf{A}_{\mathbf{1}}\right)$ and $\left(\mathbf{A}_{\mathbf{2}}\right)$, Eq. (1.1) has a variational structure.

Lemma 2.1. Suppose that $p, b, s$ and $q$ satisfy (1.2) and (1.3) and $V$ and $K$ satisfy $\left(\mathbf{A}_{\mathbf{1}}\right)$ and $\left(\mathbf{A}_{\mathbf{2}}\right)$. Then there exists $C>0$ such that the following inequality holds

$$
\begin{aligned}
& \left(\int_{\mathbb{R}^{N}} K(x)|u|^{q} d x\right)^{p / q} \leq C\left(\int_{\mathbb{R}^{N}}|\nabla u|^{p} d x+\int_{\mathbb{R}^{N}} V(x)|u|^{p} d x\right), \\
& \forall u \in C_{0}^{\infty}\left(\mathbb{R}^{N}\right) .
\end{aligned}
$$

Proof. Let

$$
\chi(x):= \begin{cases}1, & |x| \leq L \\ 0, & |x|>L\end{cases}
$$


$V_{1}=V+\chi$ and $K_{1}=K+\chi$. By $\left(\mathbf{A}_{1}\right)$ and $\left(\mathbf{A}_{2}\right)$, there exist positive constants $C^{\prime \prime}$ and $C^{\prime}$ such that for all $x \in \mathbb{R}^{N}$,

$$
\begin{aligned}
& C^{\prime}(1+|x|)^{-b} \leq V_{1}(x) \leq C^{\prime \prime}(1+|x|)^{-b}, \\
& C^{\prime}(1+|x|)^{-s} \leq K_{1}(x) \leq C^{\prime \prime}(1+|x|)^{-s} .
\end{aligned}
$$

By (2.2), the Hölder and the Sobolev inequalities (see, e.g., [28, Theorem 1.8]), we have, for every $u \in C_{0}^{\infty}\left(\mathbb{R}^{N}\right)$,

$$
\begin{aligned}
\left(\int_{\mathbb{R}^{N}} K_{1}(x)|u|^{q} d x\right)^{\frac{1}{q}} \leq & \left.C\left(\int_{\mathbb{R}^{N}} \frac{|u|^{q}}{(1+|x|)^{s}} d x\right)^{\frac{1}{q}} \quad \text { (here we used }(2.2)\right) \\
= & C\left(\int_{\mathbb{R}^{N}} \frac{|u|^{\frac{p s}{b}}}{(1+|x|)^{s}} \cdot|u|^{q-\frac{p s}{b}} d x\right)^{\frac{1}{q}} \\
\leq & C\left(\int_{\mathbb{R}^{N}} \frac{|u|^{p}}{(1+|x|)^{b}} d x\right)^{\frac{s}{q b}}\left(\int_{\mathbb{R}^{N}}|u|^{p^{*}} d x\right)^{\frac{1}{q}\left(1-\frac{s}{b}\right)} \\
& \left(\text { here we used }\left(1-\frac{s}{b}\right)^{-1}\left(q-\frac{p s}{b}\right)=p^{*}\right) \\
\leq & C\left(\int_{\mathbb{R}^{N}} \frac{|u|^{p}}{(1+|x|)^{b}} d x\right)^{\frac{s}{q b}}\left(\int_{\mathbb{R}^{N}}|\nabla u|^{p} d x\right)^{\frac{p^{*}}{q p}\left(1-\frac{s}{b}\right)} \\
= & C\left(\int_{\mathbb{R}^{N}} \frac{|u|^{p}}{(1+|x|)^{b}} d x\right)^{\frac{1}{p} \cdot \frac{p s}{q b}}\left(\int_{\mathbb{R}^{N}}|\nabla u|^{p} d x\right)^{\frac{1}{p} \cdot\left(1-\frac{p s}{q b}\right)} \\
& \left(\text { here we used } \frac{p^{*}}{q}\left(1-\frac{s}{b}\right)=1-\frac{p s}{b q}\right) \\
\leq & C\left(\int_{\mathbb{R}^{N}} V_{1}(x)|u|^{p} d x\right)^{\frac{1}{p} \cdot \frac{p s}{q b}}\left(\int_{\mathbb{R}^{N}}|\nabla u|^{p} d x\right)^{\frac{1}{p} \cdot\left(1-\frac{p s}{q b}\right)},
\end{aligned}
$$

(here we used (2.2))

where $C$ is a positive constant independent of $u$. It follows that there exists a constant $C>0$ such that

$$
\begin{aligned}
\left(\int_{\mathbb{R}^{N}} K|u|^{q} d x\right)^{1 / q} & \leq\left(\int_{\mathbb{R}^{N}} K_{1}|u|^{q} d x\right)^{1 / q} \\
& \leq C\left(\int_{\mathbb{R}^{N}}|\nabla u|^{p} d x\right)^{1 / p}+C\left(\int_{\mathbb{R}^{N}} V_{1}|u|^{p} d x\right)^{1 / p} .
\end{aligned}
$$

Moreover, by the Sobolev inequality, we have, for any $u \in C_{0}^{\infty}\left(\mathbb{R}^{N}\right)$,

$$
\begin{aligned}
\int_{\mathbb{R}^{N}} \chi(x)|u|^{p} d x & \leq\left(\int_{\mathbb{R}^{N}} \chi^{\frac{p^{*}}{p^{*}-p}}\right)^{1-\frac{p}{p^{*}}}\left(\int_{\mathbb{R}^{N}}|u|^{p^{*}}\right)^{\frac{p}{p^{*}}} \\
& \leq C L^{N\left(1-\frac{p}{p^{*}}\right)}\left(\int_{\mathbb{R}^{N}}|\nabla u|^{p}\right)
\end{aligned}
$$

where $C=C(N, p)>0$ is a constant which depends only on $N$ and $p$. Inequality (2.1) follows from (2.4) and (2.5) immediately. 
Remark 2.2. Inequality (2.1) is a weighted Sobolev inequality. For more knowledge of weighted Sobolev inequalities, one can consult the Opic and Kufner's book [17].

Let $E$ be the completion of $C_{0}^{\infty}\left(\mathbb{R}^{N}\right)$ with respect to the norm

$$
\|u\|_{E}=\left(\int_{\mathbb{R}^{N}}|\nabla u|^{p} d x+\int_{\mathbb{R}^{N}} V(x)|u|^{p} d x\right)^{1 / p} .
$$

From [1], it is easy to verify that under this norm, $E$ is an uniformly convex Banach space. By Lemma 2.1, the functional

$$
\Phi(u)=\frac{1}{p}\|u\|_{E}^{p}-\frac{1}{q} \int_{\mathbb{R}^{N}} K(x)|u|^{q} d x, \quad u \in E
$$

is well defined in $E$. It is easy to check that $\Phi$ is a $C^{1}$ functional and the derivative of $\Phi$ is given by

$$
\begin{aligned}
\left\langle\Phi^{\prime}(u), v\right\rangle= & \int_{\mathbb{R}^{N}}|\nabla u|^{p-2} \nabla u \nabla v d x+\int_{\mathbb{R}^{N}} V(x)|u|^{p-2} u v d x \\
& -\int_{\mathbb{R}^{N}} K(x)|u|^{q-2} u v d x, \quad \forall u, v \in E .
\end{aligned}
$$

It follows that the critical points of $\Phi$ are weak solutions of (1.1).

\section{Palais-Smale condition for the functional $\Phi$}

Let

$$
S=\inf _{u \in E \backslash\{0\}} \frac{\int_{\mathbb{R}^{N}}|\nabla u|^{p} d x+\int_{\mathbb{R}^{N}} V(x)|u|^{p} d x}{\left(\int_{\mathbb{R}^{N}} K(x)|u|^{q} d x\right)^{p / q}} .
$$

For $R>L$, let $(B(0, R))^{c}=\left\{x \in \mathbb{R}^{N}|| x \mid>R\right\}$ and

$$
S_{R}:=\inf _{u \in C_{0}^{\infty}\left((B(0, R))^{c}\right) \backslash\{0\}} \frac{\int_{(B(0, R))^{c}}|\nabla u|^{p} d x+\int_{(B(0, R))^{c}} V(x)|u|^{p} d x}{\left(\int_{(B(0, R))^{c}} K(x)|u|^{q} d x\right)^{p / q}} .
$$

Let

$$
S_{\infty}:=\lim _{R \rightarrow \infty} S_{R}
$$

Lemma 3.1. Suppose that $p, b, s$ and $q$ satisfy (1.2) and (1.3) and $V$ and $K$ satisfy $\left(\mathbf{A}_{\mathbf{1}}\right)$ and $\left(\mathbf{A}_{\mathbf{2}}\right)$. Then

$$
S_{\infty} \geq C_{1}^{1-\frac{p s}{q b}} D_{2}^{-\frac{p}{q}}\left(\max \left\{\frac{q b}{p s},\left(1-\frac{p s}{q b}\right)^{-1}\right\}\right)^{-1} \cdot A
$$

where

$$
A=\inf _{u \in C_{0}^{\infty}\left((B(0, L))^{c}\right) \backslash\{0\}} \frac{\left(\int_{(B(0, L))^{c}}|\nabla u|^{p} d x\right)^{\frac{p s}{q b}}\left(\int_{(B(0, L))^{c}}|x|^{-b}|u|^{p} d x\right)^{1-\frac{p s}{q b}}}{\left(\int_{(B(0, L))^{c}}|x|^{-s}|u|^{q} d x\right)^{p / q}} .
$$


Proof. By assumptions $\left(\mathbf{A}_{\mathbf{1}}\right)$ and $\left(\mathbf{A}_{\mathbf{2}}\right)$, for $|x| \geq L$,

$$
V(x) \geq \frac{C_{1}}{|x|^{b}}, \text { and } K(x) \leq \frac{D_{2}}{|x|^{s}} .
$$

This together with (3.2) and (3.3) implies that

$$
S_{\infty} \geq \lim _{R \rightarrow \infty u \in C_{0}^{\infty}\left((B(0, R))^{c}\right) \backslash\{0\}} \frac{\int_{(B(0, R))^{c}}|\nabla u|^{p} d x+C_{1} \int_{(B(0, R))^{c}}|x|^{-b}|u|^{p} d x}{\left(D_{2} \int_{(B(0, R))^{c}}|x|^{-s}|u|^{q} d x\right)^{p / q}} .
$$

From (2.3), we deduce that for $R \geq L$ and $u \in C_{0}^{\infty}\left((B(0, R))^{c}\right)$,

$$
\begin{aligned}
& \frac{q b}{p s} \int_{(B(0, R))^{c}}|\nabla u|^{p} d x+\left(1-\frac{p s}{q b}\right)^{-1} \int_{(B(0, R))^{c}} C_{1}|x|^{-b}|u|^{p} d x \\
& \geq C_{1}^{1-\frac{p s}{q b}}\left(\int_{(B(0, R))^{c}}|\nabla u|^{p} d x\right)^{\frac{p s}{q b}}\left(\int_{(B(0, R))^{c}}|x|^{-b}|u|^{p} d x\right)^{1-\frac{p s}{q b}} \\
& \geq A_{R} C_{1}^{1-\frac{p s}{q b}}\left(\int_{(B(0, R))^{c}}|x|^{-s}|u|^{q} d x\right)^{p / q}
\end{aligned}
$$

where

$$
A_{R}=\inf _{u \in C_{0}^{\infty}\left((B(0, R))^{c}\right) \backslash\{0\}} \frac{\left(\int_{(B(0, R))^{c}}|\nabla u|^{p} d x\right)^{\frac{p s}{q b}}\left(\int_{(B(0, R))^{c}}|x|^{-b}|u|^{p} d x\right)^{1-\frac{p s}{q b}}}{\left(\int_{(B(0, R))^{c}}|x|^{-s}|u|^{q} d x\right)^{p / q}} \geq A .
$$

From (3.6) and $A_{R} \geq A$, we have that, for any $R \geq L$ and $u \in C_{0}^{\infty}\left((B(0, R))^{c}\right)$,

$$
\begin{gathered}
\max \left\{\frac{q b}{p s},\left(1-\frac{p s}{q b}\right)^{-1}\right\} \cdot\left(\int_{(B(0, R))^{c}}|\nabla u|^{p} d x+\int_{(B(0, R))^{c}} C_{1}|x|^{-b}|u|^{p} d x\right) \\
\geq A C_{1}^{1-\frac{p s}{q b}}\left(\int_{(B(0, R))^{c}}|x|^{-s}|u|^{q} d x\right)^{p / q} .
\end{gathered}
$$

Inequality (3.4) follows from this inequality and (3.5).

Definition 3.2. Let $\phi \in C^{1}(E, \mathbb{R})$. A sequence $\left\{u_{n}\right\} \subset E$ is called a PalaisSmale sequence at level $c\left((P S)_{c}\right.$ sequence for short) for $\phi$, if $\phi\left(u_{n}\right) \rightarrow c$ and $\left\|\phi^{\prime}\left(u_{n}\right)\right\|_{E^{\prime}} \rightarrow 0$ as $n \rightarrow \infty$. $\phi$ is said to satisfy $(P S)_{c}$ condition if every $(P S)_{c}$ sequence of $\phi$ contains a convergent subsequence in $E$.

Theorem 3.3. For $c<\left(\frac{1}{p}-\frac{1}{q}\right) S_{\infty}^{\frac{q}{q-p}}$, the functional $\Phi$, defined by (2.6), satisfies the $(P S)_{c}$ condition.

Proof. Let $\left\{u_{n}\right\} \subset E$ be a $(P S)_{c}$ sequence for $\Phi$, i.e., $\Phi\left(u_{n}\right) \rightarrow c$ and $\Phi^{\prime}\left(u_{n}\right) \rightarrow$ 0 in $E^{\prime}$. Then

$$
\left\|u_{n}\right\|_{E}^{p}=\left(\frac{q}{p}-1\right)^{-1}\left(q \Phi\left(u_{n}\right)-\left\langle\Phi^{\prime}\left(u_{n}\right), u_{n}\right\rangle\right)=\frac{p q c}{q-p}+o(1) \cdot\left\|u_{n}\right\|_{E},
$$


where $o(1)$ denotes the infinitesimal depending only on $n$, i.e., $o(1) \rightarrow 0$ as $n \rightarrow \infty$. It follows that

$$
\sup _{n}\left\|u_{n}\right\|_{E}<+\infty
$$

Then

$$
\begin{aligned}
\int_{\mathbb{R}^{N}} K(x)\left|u_{n}\right|^{q} d x & =\left(\frac{1}{p}-\frac{1}{q}\right)^{-1}\left(\Phi\left(u_{n}\right)-\frac{1}{p}\left\langle\Phi^{\prime}\left(u_{n}\right), u_{n}\right\rangle\right) \\
& =\left(\frac{1}{p}-\frac{1}{q}\right)^{-1} c+o(1) .
\end{aligned}
$$

Together with the assumption $c<\left(\frac{1}{p}-\frac{1}{q}\right) S_{\infty}^{\frac{q}{q-p}}$ and the fact that $\lim _{R \rightarrow \infty} S_{R}=$ $S_{\infty}$, this implies that there exist positive constants $R_{0}, \delta_{0} \in(0,1)$ and $m_{0}$ such that for $n \geq m_{0}$ and $R \geq R_{0}$,

$$
1-S_{R}^{-1}\left(\int_{\mathbb{R}^{N}} K(x)\left|u_{n}\right|^{q} d x\right)^{\frac{q-p}{q}} \geq 2 \delta_{0} .
$$

Let $\eta \in C^{\infty}[0,+\infty)$ be such that

$$
0 \leq \eta \leq 1 ; \eta(r)=0, r \in[0,1] ; \eta(r)=1, r \in[2,+\infty) ; 0 \leq \eta^{\prime}(r) \leq 2 .
$$

For $R>0$ and $x \in \mathbb{R}^{N}$, let $\eta_{R}(x)=\eta(|x| / R)$. Then

$$
\left|\nabla \eta_{R}(x)\right| \leq 2 / R, \quad \forall x \in \mathbb{R}^{N}
$$

By (1.4) and (3.9), for $R \geq R_{0}$,

$$
\begin{aligned}
\int_{\mathbb{R}^{N}}\left|\nabla\left(\eta_{R}^{p} u_{n}\right)\right|^{p} d x & \leq C \int_{\mathbb{R}^{N}} \eta_{R}^{p^{2}}\left|\nabla u_{n}\right|^{p} d x+C \int_{\mathbb{R}^{N}}\left|u_{n}\right|^{p}\left|\nabla \eta_{R}^{p}\right|^{p} d x \\
& \leq C \int_{\mathbb{R}^{N}}\left|\nabla u_{n}\right|^{p} d x+\frac{C}{R^{p}} \int_{R \leq|x| \leq 2 R}\left|u_{n}\right|^{p} d x \\
& \leq C \int_{\mathbb{R}^{N}}\left|\nabla u_{n}\right|^{p} d x+\frac{C}{R^{p-b}} \int_{R \leq|x| \leq 2 R} V(x)\left|u_{n}\right|^{p} d x \\
& \left.\leq C|| u_{n} \|_{E}^{p} . \quad \text { (here we used } p-b>0\right) .
\end{aligned}
$$

Together with (3.7) and (3.10), this yields

$$
\sup \left\{\left\|\eta_{R}^{p} u_{n}\right\|_{E} \mid R \geq R_{0}, n \geq 1\right\}<+\infty .
$$

Then, by $\Phi^{\prime}\left(u_{n}\right) \rightarrow 0$ in $E^{\prime}$, we obtain $\left\langle\Phi^{\prime}\left(u_{n}\right), \eta_{R}^{p} u_{n}\right\rangle=o(1)$, i.e.,

$$
\begin{gathered}
\int_{\mathbb{R}^{N}}\left|\nabla u_{n}\right|^{p-2} \nabla u_{n} \nabla\left(\eta_{R}^{p} u_{n}\right) d x+\int_{\mathbb{R}^{N}} V(x)\left|\eta_{R} u_{n}\right|^{p} \\
=\int_{\mathbb{R}^{N}} K(x)\left|u_{n}\right|^{q-2} u_{n} \cdot\left(\eta_{R}^{p} u_{n}\right) d x+o(1) .
\end{gathered}
$$

By the Hölder inequality, for any $\epsilon>0$, there exists $C_{\epsilon}>0$ such that

$$
\left.\left.\left|u_{n} \eta_{R}^{p-1}\right| \nabla u_{n}\right|^{p-2} \nabla u_{n} \nabla \eta_{R}\left|\leq \epsilon \eta_{R}^{p}\right| \nabla u_{n}\right|^{p}+C_{\epsilon}\left|u_{n}\right|^{p}\left|\nabla \eta_{R}\right|^{p} .
$$


Using this inequality with $\epsilon=\delta_{0} / 2 p$, we get that there exists a positive constant $C>0$ such that

$$
\begin{aligned}
& \int_{\mathbb{R}^{N}}\left|\nabla u_{n}\right|^{p-2} \nabla u_{n} \nabla\left(\eta_{R}^{p} u_{n}\right) d x \\
& \quad=\int_{\mathbb{R}^{N}}\left|\nabla u_{n}\right|^{p} \eta_{R}^{p} d x+p \int_{\mathbb{R}^{N}} u_{n} \eta_{R}^{p-1}\left|\nabla u_{n}\right|^{p-2} \nabla u_{n} \nabla \eta_{R} d x \\
& \quad \geq\left(1-\frac{\delta_{0}}{2}\right) \int_{\mathbb{R}^{N}}\left|\nabla u_{n}\right|^{p} \eta_{R}^{p} d x-C \int_{\mathbb{R}^{N}}\left|u_{n}\right|^{p}\left|\nabla \eta_{R}\right|^{p} d x
\end{aligned}
$$

By the mean value theorem, we have

$$
\begin{aligned}
& \left|\eta_{R} \nabla u_{n}\right|^{p}-\left|\nabla\left(\eta_{R} u_{n}\right)\right|^{p} \\
& \quad=p\left((1-\theta)\left|\eta_{R} \nabla u_{n}\right|+\theta\left|\nabla\left(\eta_{R} u_{n}\right)\right|\right)^{p-1}\left(\left|\eta_{R} \nabla u_{n}\right|-\left|\nabla\left(\eta_{R} u_{n}\right)\right|\right) \\
& \quad \geq-p\left(\left|\eta_{R} \nabla u_{n}\right|+\left|u_{n} \nabla \eta_{R}\right|\right)^{p-1}\left|u_{n} \nabla \eta_{R}\right|,
\end{aligned}
$$

where $0<\theta=\theta(x)<1, x \in \mathbb{R}^{N}$. By the Hölder inequality, for any $\epsilon>0$, there exists $C_{\epsilon}>0$ such that

$$
\begin{aligned}
\left(\left|\eta_{R} \nabla u_{n}\right|+\left|u_{n} \nabla \eta_{R}\right|\right)^{p-1}\left|u_{n} \nabla \eta_{R}\right| & \leq \epsilon\left(\left|\eta_{R} \nabla u_{n}\right|+\left|u_{n} \nabla \eta_{R}\right|\right)^{p}+C_{\epsilon}\left|u_{n} \nabla \eta_{R}\right|^{p} \\
& \leq C_{p} \epsilon\left|\eta_{R} \nabla u_{n}\right|^{p}+\left(C_{p} \epsilon+C_{\epsilon}\right)\left|u_{n} \nabla \eta_{R}\right|^{p}
\end{aligned}
$$

where $C_{p}>0$ depends only on $p$. Choosing $\epsilon=\delta_{0} / 2\left(1-\delta_{0}\right) p C_{p}$ in (3.14) and combining (3.13) with (3.14), we get

$$
\left|\eta_{R} \nabla u_{n}\right|^{p} \geq \frac{2\left(1-\delta_{0}\right)}{\left(2-\delta_{0}\right)}\left|\nabla\left(\eta_{R} u_{n}\right)\right|^{p}-C\left|u_{n} \nabla \eta_{R}\right|^{p}
$$

Combining (3.12) and (3.15) leads to

$$
\begin{aligned}
\int_{\mathbb{R}^{N}}\left|\nabla u_{n}\right|^{p-2} \nabla u_{n} \nabla\left(\eta_{R}^{p} u_{n}\right) d x \geq & \left(1-\delta_{0}\right) \int_{\mathbb{R}^{N}}\left|\nabla\left(\eta_{R} u_{n}\right)\right|^{p} d x \\
& -\frac{C}{R^{p}} \int_{R \leq|x| \leq 2 R}\left|u_{n}\right|^{p} d x,
\end{aligned}
$$

where $C$ is a positive constant which is independent of $n$ and $R$. From (1.4) and (3.7), we have

$$
\frac{1}{R^{p}} \int_{R \leq|x| \leq 2 R}\left|u_{n}\right|^{p} d x \leq \frac{C}{R^{p-b}} \int_{R \leq|x| \leq 2 R} V(x)\left|u_{n}\right|^{p} d x \leq \frac{C}{R^{p-b}}
$$

Combining (3.16) with (3.17) yields that there exists $C>0$ which is independent of $n$ and $R$ such that

$$
\int_{\mathbb{R}^{N}}\left|\nabla u_{n}\right|^{p-2} \nabla u_{n} \nabla\left(\eta_{R}^{p} u_{n}\right) d x \geq\left(1-\delta_{0}\right) \int_{\mathbb{R}^{N}}\left|\nabla\left(\eta_{R} u_{n}\right)\right|^{p} d x-\frac{C}{R^{p-b}} .
$$

Together with (3.11), this implies

$$
\int_{\mathbb{R}^{N}}\left|\nabla u_{n}\right|^{p-2} \nabla u_{n} \nabla\left(\eta_{R}^{p} u_{n}\right) d x+\int_{\mathbb{R}^{N}} V(x)\left|\eta_{R} u_{n}\right|^{p} \geq\left.\left(1-\delta_{0}\right)|| \eta_{R} u_{n}\right|_{E} ^{p}-\frac{C}{R^{p-b}} .
$$


Combining (3.19), (3.11), (3.8) and the following inequality

$$
\begin{aligned}
& \int_{\mathbb{R}^{N}} K(x)\left|u_{n}\right|^{q-2} u_{n} \cdot\left(\eta_{R}^{p} u_{n}\right) d x \\
& \leq\left(\int_{|x| \geq R} K(x)\left|u_{n}\right|^{q}\right)^{\frac{q-p}{q}}\left(\int_{\mathbb{R}^{N}} K(x)\left|\eta_{R} u_{n}\right|^{q}\right)^{\frac{p}{q}} \\
& \leq\left. S_{R}^{-1}\left(\int_{|x| \geq R} K(x)\left|u_{n}\right|^{q}\right)^{\frac{q-p}{q}}|| \eta_{R} u_{n}\right|_{E} ^{p},
\end{aligned}
$$

we obtain

$$
\delta_{0}\left\|\eta_{R} u_{n}\right\|_{E}^{p} \leq \frac{C}{R^{p-b}}+o(1) .
$$

Together with (3.1), this yields

$$
\delta_{0} S\left(\int_{\mathbb{R}^{N}} K(x)\left|\eta_{R} u_{n}\right|^{q} d x\right)^{\frac{p}{q}} \leq \frac{C}{R^{p-b}}+o(1) .
$$

Since $E$ is a reflexive Banach space and $\left\{\left\|u_{n}\right\|_{E}\right\}$ is bounded in $E$, we deduce that there exists $u_{0} \in E$ such that, up to a subsequence, $u_{n} \rightarrow u_{0}$ in $E$. It is easy to verify that $u_{0}$ is a critical point of $\Phi$.

By (3.22), we have

$$
\begin{aligned}
& \left.\left|\int_{\mathbb{R}^{N}} K(x)\right| u_{n}\right|^{q} d x-\int_{\mathbb{R}^{N}} K(x)\left|u_{0}\right|^{q} d x \mid \\
& \leq\left.\left|\int_{\mathbb{R}^{N}} K(x)\right|\left(1-\eta_{R}\right) u_{n}\right|^{q} d x-\int_{\mathbb{R}^{N}} K(x)\left|\left(1-\eta_{R}\right) u_{0}\right|^{q} d x \mid \\
& \quad+\int_{\mathbb{R}^{N}} K(x)\left|\eta_{R} u_{n}\right|^{q} d x+\int_{\mathbb{R}^{N}} K(x)\left|\eta_{R} u_{0}\right|^{q} d x \\
& \leq\left.\left|\int_{\mathbb{R}^{N}} K(x)\right|\left(1-\eta_{R}\right) u_{n}\right|^{q} d x-\int_{\mathbb{R}^{N}} K(x)\left|\left(1-\eta_{R}\right) u_{0}\right|^{q} d x \mid \\
& \quad+\frac{C}{R^{\frac{q}{p}(p-b)}}+\int_{\mathbb{R}^{N}} K(x)\left|\eta_{R} u_{0}\right|^{q} d x+o(1) .
\end{aligned}
$$

Since the imbedding $E \hookrightarrow L_{l o c}^{p}\left(\mathbb{R}^{N}\right)$ is compact, we get

$$
\left.\lim _{n \rightarrow \infty}\left|\int_{\mathbb{R}^{N}} K(x)\right|\left(1-\eta_{R}\right) u_{n}\right|^{q} d x-\int_{\mathbb{R}^{N}} K(x)\left|\left(1-\eta_{R}\right) u_{0}\right|^{q} d x \mid=0 .
$$

Then, by (3.23), we obtain that

$$
\begin{aligned}
& \left.\limsup _{n \rightarrow \infty}\left|\int_{\mathbb{R}^{N}} K(x)\right| u_{n}\right|^{q} d x-\int_{\mathbb{R}^{N}} K(x)\left|u_{0}\right|^{q} d x \mid \\
& \quad \leq \frac{C}{R^{\frac{q}{p}(p-b)}}+\int_{\mathbb{R}^{N}} K(x)\left|\eta_{R} u_{0}\right|^{q} d x .
\end{aligned}
$$

Together with $C R^{-\frac{q}{p}(p-b)}+\int_{\mathbb{R}^{N}} K(x)\left|\eta_{R} u_{0}\right|^{q} d x \rightarrow 0$ as $R \rightarrow \infty$, this implies

$$
\left.\limsup _{n \rightarrow \infty}\left|\int_{\mathbb{R}^{N}} K(x)\right| u_{n}\right|^{q} d x-\int_{\mathbb{R}^{N}} K(x)\left|u_{0}\right|^{q} d x \mid=0 .
$$


By $\left\langle\Phi^{\prime}\left(u_{n}\right), u_{n}\right\rangle=o(1)$ and $\left\langle\Phi^{\prime}\left(u_{0}\right), u_{0}\right\rangle=0$, we have

$$
\left\|u_{n}\right\|_{E}^{p}=\int_{\mathbb{R}^{N}} K(x)\left|u_{n}\right|^{q} d x+o(1),\left\|u_{0}\right\|_{E}^{p}=\int_{\mathbb{R}^{N}} K(x)\left|u_{0}\right|^{q} d x .
$$

Together with (3.24), we get $\lim _{n \rightarrow \infty}\left\|u_{n}\right\|_{E}=\left\|u_{0}\right\|_{E}$. Finally, by the fact that $E$ is uniformly convex Banach space, we deduce that $u_{n} \rightarrow u_{0}$ in $E$. This completes the proof.

Theorem 3.4. If $S<S_{\infty}$, then the infimum $S$ is achieved.

Proof. Let $\left\{u_{n}\right\}$ be a minimizing sequence. We assume $\int_{\mathbb{R}^{N}} K(x)\left|u_{n}\right|^{q} d x=1$, $n=1,2, \ldots$, otherwise, we choose $u_{n} /\left(\int_{\mathbb{R}^{N}} K(x)\left|u_{n}\right|^{q} d x\right)^{1 / q}$ as the minimizing sequence. Then

$$
\left\|u_{n}\right\|_{E}^{p} \rightarrow S, \int_{\mathbb{R}^{N}} K(x)\left|u_{n}\right|^{q} d x=1, \quad n=1,2, \ldots
$$

By the Ekeland variational principle (see, e.g., [28]) and the Lagrange multiplier rule, we have

$$
\begin{aligned}
& \int_{\mathbb{R}^{N}}\left|\nabla u_{n}\right|^{p-2} \nabla u_{n} \nabla \varphi d x+\int_{\mathbb{R}^{N}} V(x)\left|u_{n}\right|^{p-2} u_{n} \varphi d x \\
& \quad-S \int_{\mathbb{R}^{N}} K(x)\left|u_{n}\right|^{q-2} u_{n} \varphi d x \rightarrow 0
\end{aligned}
$$

holds uniformly for $\varphi \in E$ with $\|\varphi\|_{E} \leq 1$. Let $v_{n}=S^{\frac{1}{q-p}} u_{n}$. Then by (3.25) and $(3.26)$, we deduce that $\left\{v_{n}\right\}$ is a $(P S)_{c}$ sequence for $\Phi$ with $c=\left(\frac{1}{p}-\right.$ $\left.\frac{1}{q}\right) S^{\frac{q}{q-p}}$. Since $S<S_{\infty}$, by Theorem 3.3, there exists $v \in E$ such that, up to a subsequence, $v_{n} \rightarrow v$ in $E$. It follows that $u_{n} \rightarrow u=S^{-\frac{1}{q-p}} v$. Then $u$ achieves the infimum $S$.

Remark 3.5. When $p=2$, the recent paper [11] provides some conditions on the potentials $V$ and $K$ ensuring that the inequality $S<S_{\infty}$ is satisfied (see Theorem 1.1 of [11]). In Sect. 4 we consider the case of a steep potential well and give conditions that also ensure the inequality $S<S_{\infty}$.

\section{Equation (1.1) with a steep potential well and a critical frequency}

In this section, we assume that $V$ and $K$ satisfy the additional assumptions

(V). There exists a closed subset $Z \subset\left\{x \in \mathbb{R}^{N}|| x \mid<L\right\}$ with nonempty interior $\Omega=\operatorname{int} Z$ such that $V(x)=0$ for $x \in Z$.

$(\mathbf{K}) \cdot \inf _{|x| \leq L} K(x)>0$.

Consider the equation

$$
-\Delta_{p} u+\lambda V(x)|u|^{p-2} u=K(x)|u|^{q-2} u, \quad u \in E_{\lambda}
$$

where $\lambda$ is a positive parameter and $E_{\lambda}$ is the Banach space $E$ defined in Sect. 2 with $V$ replaced by $\lambda V$. The norm of $E_{\lambda}$ is denoted by $\|\cdot\|_{\lambda}$. The functional corresponding to this equation is denoted by $\Phi_{\lambda}$ which is the functional defined 
by (2.6) with $\|u\|_{E}^{p}$ replaced by $\|u\|_{\lambda}^{p}$. Let $S_{\lambda}$ and $S_{R}^{\lambda}$ be $S$ and $S_{R}$ with $V$ replaced by $\lambda V$ and let $S_{\infty}^{\lambda}=\lim _{R \rightarrow \infty} S_{R}^{\lambda}$. By (3.4), we have

$$
S_{\infty}^{\lambda} \geq\left(\lambda C_{1}\right)^{1-\frac{p s}{q b}} D_{2}^{-\frac{p}{q}}\left(\max \left\{\frac{q b}{p s},\left(1-\frac{p s}{q b}\right)^{-1}\right\}\right)^{-1} \cdot A .
$$

This implies

$$
\lim _{\lambda \rightarrow+\infty} S_{\infty}^{\lambda}=+\infty
$$

On the other hand, choose a function $v_{0} \in C_{0}^{\infty}\left(\mathbb{R}^{N}\right)$ satisfying $v_{0} \neq 0$ and the support of $v_{0}$ is contained in $Z$. Then by $(\mathbf{V})$ and $(\mathbf{K})$, we get that, for every $\lambda>0$,

$$
S_{\lambda} \leq \frac{\int_{\mathbb{R}^{N}}\left|\nabla v_{0}\right|^{p} d x}{\left(\int_{\mathbb{R}^{N}} K(x)\left|v_{0}\right|^{q} d x\right)^{p / q}} .
$$

Together with (4.2) and Theorem 3.4, this leads to the following corollary

Corollary 4.1. Suppose that $\left(\mathbf{A}_{\mathbf{1}}\right),\left(\mathbf{A}_{\mathbf{2}}\right),(\mathbf{V})$ and $(\mathbf{K})$ hold. Then there exists $\Lambda^{*}>0$, such that for $\lambda \geq \Lambda^{*}$, the infimum $S_{\lambda}$ is achieved.

Theorem 4.2. Suppose that $\left(\mathbf{A}_{\mathbf{1}}\right),\left(\mathbf{A}_{\mathbf{2}}\right),(\mathbf{V})$ and $(\mathbf{K})$ hold. Then for every integer $m$ there exists $\Lambda_{m} \geq 1$ such that (4.1) with $\lambda \geq \Lambda_{m}$ has at least $m$ distinct pairs $\pm v_{1}^{\lambda}, \ldots, \pm v_{m}^{\lambda}$ of weak solutions. Moreover, there exist positive constants $a, b_{m}$ (independent of $\lambda$ ) such that

$$
a \leq\left\|v_{j}^{\lambda}\right\|_{\lambda} \leq b_{m}, 1 \leq j \leq m
$$

hold for all $\lambda \geq \Lambda_{m}$.

Proof. Let $W_{0}^{1, p}(\Omega)=\left\{\left.u\left|\int_{\Omega}\right| \nabla u\right|^{p} d x<+\infty,\left.u\right|_{\partial \Omega}=0\right\}$. By $(\mathbf{V}), W_{0}^{1, p}(\Omega) \subset$ $E_{\lambda}$ for all $\lambda>0$. Let

$$
\Phi_{0}(u)=\frac{1}{p} \int_{\Omega}|\nabla u|^{p} d x-\frac{1}{q} \int_{\Omega} K(x)|u|^{q} d x, u \in W_{0}^{1, p}(\Omega) .
$$

If $0 \neq u \in E_{\lambda}$ is a critical point of $\Phi_{\lambda}$, by $\left\langle\Phi_{\lambda}^{\prime}(u), u\right\rangle=0, S \leq S_{\lambda}(\lambda \geq 1)$ and Lemma 2.1, we get that, for $\lambda \geq 1$,

$$
\|u\|_{\lambda}^{p}=\int_{\mathbb{R}^{N}} K(x)|u|^{q} d x \leq S_{\lambda}^{-\frac{q}{p}}\|u\|_{\lambda}^{q} \leq S^{-\frac{q}{p}}\|u\|_{\lambda}^{q} .
$$

It follows that for $\lambda \geq 1$,

$$
\|u\|_{\lambda} \geq S^{q / p(q-p)}:=a .
$$

Let $\left\{e_{n}\right\} \subset W_{0}^{1, p}(\Omega)$ be a linearly independent sequence and $E_{j}:=$ $\operatorname{span}\left\{e_{1}, \ldots, e_{j}\right\}$. By $(\mathbf{V}), \Phi_{\lambda}(u)=\Phi_{0}(u), \forall u \in W_{0}^{1, p}(\Omega)$. Then by $(\mathbf{K})$ and $q>p$, we deduce that there exists an increasing sequence of positive numbers $\left\{R_{j}\right\}$ (independent of $\lambda$ ) such that

$$
\Phi_{\lambda}(u) \leq 0, \forall u \in E_{j},\|u\|_{\lambda} \geq R_{j} .
$$


Let $D_{j}=\left\{u \mid u \in E_{j},\|u\|_{\lambda} \leq R_{j}\right\}$ and $\partial D_{j}$ be the boundary of $D_{j}$ in $E_{j}$. Let $\Sigma$ be the class of closed symmetric subsets of $E_{\lambda}$ and $\gamma(Y)$ be the genus of $Y$ for $Y \in \Sigma$ (see [18] for its definition). Define for $j=1,2, \ldots$,

$$
\begin{aligned}
& G_{j}=\left\{h \mid h \in C\left(D_{j}, E_{\lambda}\right), h \text { is odd, }\left.h\right|_{\partial D_{j}}=i d\right\}, \\
& \widetilde{G}_{j}=\left\{h \mid h \in C\left(D_{j}, W_{0}^{1, p}(\Omega)\right), h \text { is odd, }\left.h\right|_{\partial D_{j}}=i d\right\}, \\
& \Gamma_{j}=\left\{h\left(\overline{D_{k} \backslash Y}\right) \mid h \in G_{k}, k \geq j, Y \in \Sigma, \gamma(Y) \leq k-j\right\}, \\
& \widetilde{\Gamma}_{j}=\left\{h\left(\overline{D_{k} \backslash Y}\right) \mid h \in \widetilde{G}_{k}, k \geq j, \quad Y \in \Sigma, \gamma(Y) \leq k-j\right\},
\end{aligned}
$$

where $\bar{F}$ denotes the closure of a set $F$. It follows that $\widetilde{\Gamma}_{j} \subset \Gamma_{j}$ for all $j$. Define for $j=1,2, \ldots$,

$$
c_{j}^{\lambda}=\inf _{B \in \Gamma_{j}} \sup _{u \in B} \Phi_{\lambda}(u), \quad \tilde{c}_{j}=\inf _{B \in \widetilde{\Gamma}_{j}} \sup _{u \in B} \Phi_{0}(u) .
$$

We have

$$
c_{1}^{\lambda} \leq c_{2}^{\lambda} \leq \ldots, \quad \tilde{c}_{1} \leq \tilde{c}_{2} \leq \ldots
$$

By $\widetilde{\Gamma}_{j} \subset \Gamma_{j}$, we obtain

$$
c_{j}^{\lambda} \leq \tilde{c}_{j}, \quad \forall j .
$$

It easy to verify that $\Phi_{\lambda}$ and $\Phi_{0}$ satisfy the $\left(I_{1}^{\prime}\right)$ and $\left(I_{2}^{\prime}\right)$ assumptions of [18, Theorem 9.12]. By (4.2), for any $m$, there exists $\Lambda_{m}>1$ such that for $\lambda \geq \Lambda_{m}, \tilde{c}_{m}<\left(\frac{1}{p}-\frac{1}{q}\right)\left(S_{\infty}^{\lambda}\right)^{q /(q-p)}$. Then by [18, Theorem 9.12] and Theorem 3.3 , for $\lambda \geq \Lambda_{m}, c_{1}^{\lambda}, \ldots, c_{m}^{\lambda}$ are critical values of $\Phi_{\lambda}$. Let $v_{j}^{\lambda}, i=1, \ldots, m$ be critical points of $\Phi_{\lambda}$ with $\Phi_{\lambda}\left(v_{j}^{\lambda}\right)=c_{j}^{\lambda}$. Then $\left\|v_{j}^{\lambda}\right\|_{\lambda}=\left(\frac{1}{p}-\frac{1}{q}\right)^{-1 / p}\left(c_{j}^{\lambda}\right)^{1 / p} \leq$ $\left(\frac{1}{p}-\frac{1}{q}\right)^{-1 / p}\left(\tilde{c}_{m}\right)^{1 / p}:=b_{m}$. Together with (4.4), this implies (4.3).

Theorem 4.3. Suppose $\left(\mathbf{A}_{\mathbf{1}}\right),\left(\mathbf{A}_{\mathbf{2}}\right),(\mathbf{V})$ and $(\mathbf{K})$ hold. Let $u_{n} \in E_{\lambda_{n}}, n \in \mathbb{N}$ be a non-zero weak solution of (4.1) for some sequence $\lambda_{n} \rightarrow+\infty$ and suppose that

$$
\sup _{n \in \mathbb{N}}\left\|u_{n}\right\|_{\lambda_{n}}<\infty
$$

holds. Then there exists $0 \neq \bar{u} \in E$ such that $u_{n} \rightarrow \bar{u}$ along a subsequence strongly in E. Moreover, $\bar{u}$ is a weak solution of the equation

$$
-\Delta_{p} u=K(x)|u|^{q-2} u, \text { for } x \in \Omega=\operatorname{int} Z
$$

and $\bar{u}=0$ a.e. in $\mathbb{R}^{N} \backslash Z$.

Proof. We divide the proof into several steps.

1. From (4.5), we deduce that $\left\{u_{n}\right\}$ is bounded in $E$. Then, there exists $\bar{u} \in E$ such that, up to a subsequence, $u_{n} \rightarrow \bar{u}$ and by the compact embedding $E \hookrightarrow L_{l o c}^{\theta}\left(\mathbb{R}^{N}\right)$ (see for example, [1]), $u_{n} \rightarrow \bar{u}$ in $L_{l o c}^{\theta}\left(\mathbb{R}^{N}\right)$ for all $p \leq \theta<p^{*}$. It follows that $\bar{u}$ is a weak solution of (4.6). 
2. For $k \in \mathbb{N}$, let $A_{k}=\left\{x \in \mathbb{R}^{N}|| x \mid \leq k, V(x) \geq 1 / k\right\}$. From the compact embedding $E \hookrightarrow L_{l o c}^{p}\left(\mathbb{R}^{N}\right)$, we have that $\int_{A_{k}}\left|u_{n}\right|^{p} d x \rightarrow \int_{A_{k}}|\bar{u}|^{p} d x$. Then by

$$
\int_{A_{k}}\left|u_{n}\right|^{p} d x \leq k \int_{A_{k}} V(x)\left|u_{n}\right|^{p} d x \leq \frac{k}{\lambda_{n}}\left\|u_{n}\right\|_{\lambda_{n}}^{p} \rightarrow 0, \quad n \rightarrow \infty,
$$

we get $\int_{A_{k}}|\bar{u}|^{p} d x=0$. Therefore, $\bar{u}=0$ a.e. in $\cup_{k=1}^{\infty} A_{k}=\mathbb{R}^{N} \backslash Z$.

3. From $\lambda_{n} \rightarrow \infty$ and (4.2), we deduce that $S_{\infty}^{\lambda_{n}} \rightarrow+\infty$ as $n \rightarrow \infty$. Then following the same argument as (3.24), we have

$$
\left.\limsup _{n \rightarrow \infty}\left|\int_{\mathbb{R}^{N}} K(x)\right| u_{n}\right|^{q} d x-\int_{\mathbb{R}^{N}} K(x)|\bar{u}|^{q} d x \mid=0 .
$$

Together with $\|\left. u_{n}\right|_{\lambda_{n}} ^{p}=\int_{\mathbb{R}^{N}} K(x)\left|u_{n}\right|^{q} d x$ and (4.4), this implies $\int_{\mathbb{R}^{N}} K(x)|\bar{u}|^{q} d x \geq S^{q /(q-p)}$. Hence $\bar{u} \neq 0$.

4. Since $u_{n}$ is a weak solution of (4.1), we have

$$
\begin{aligned}
& \int_{\mathbb{R}^{N}}\left|\nabla u_{n}\right|^{p-2} \nabla u_{n} \nabla \bar{u} d x+\lambda_{n} \int_{\mathbb{R}^{N}} V(x)\left|u_{n}\right|^{p-2} u_{n} \bar{u} d x \\
& \quad=\int_{\mathbb{R}^{N}} K(x)\left|u_{n}\right|^{q-2} u_{n} \bar{u} d x .
\end{aligned}
$$

By $\bar{u}=0$ a.e. in $\mathbb{R}^{N} \backslash Z$ and $V=0$ in $Z$, we get

$$
\int_{\mathbb{R}^{N}}\left|\nabla u_{n}\right|^{p-2} \nabla u_{n} \nabla \bar{u} d x=\int_{\mathbb{R}^{N}} K(x)\left|u_{n}\right|^{q-2} u_{n} \bar{u} d x .
$$

Sending $n$ to infinity, we obtain

$$
\int_{\mathbb{R}^{N}}|\nabla \bar{u}|^{p} d x=\int_{\mathbb{R}^{N}} K(x)|\bar{u}|^{q} d x .
$$

5. By $\lambda_{n} \rightarrow+\infty, \lambda_{n} \int_{\mathbb{R}^{N}} V(x)\left|u_{n}\right|^{p} d x \leq\left\|u_{n}\right\|_{\lambda_{n}}^{p}$ and (4.5), we get that $\int_{\mathbb{R}^{N}} V(x)\left|u_{n}\right|^{p} d x \rightarrow 0$. Therefore, to prove $u_{n} \rightarrow \bar{u}$ in $E$, it suffices to prove $\int_{\mathbb{R}^{N}}\left|\nabla u_{n}\right|^{p} d x \rightarrow \int_{\mathbb{R}^{N}}|\nabla \bar{u}|^{p} d x$. By $\left.|| u_{n}\right|_{\lambda_{n}} ^{p}=\int_{\mathbb{R}^{N}} K(x)\left|u_{n}\right|^{q} d x$, we have

$$
\int_{\mathbb{R}^{N}}\left|\nabla u_{n}\right|^{p} d x \leq \int_{\mathbb{R}^{N}} K(x)\left|u_{n}\right|^{q} d x .
$$

Then by (4.7) and (4.8),

$$
\begin{aligned}
\limsup _{n \rightarrow \infty} \int_{\mathbb{R}^{N}}\left|\nabla u_{n}\right|^{p} d x & \leq \limsup _{n \rightarrow \infty} \int_{\mathbb{R}^{N}} K(x)\left|u_{n}\right|^{q} d x=\int_{\mathbb{R}^{N}} K(x)|\bar{u}|^{q} d x \\
& =\int_{\mathbb{R}^{N}}|\nabla \bar{u}|^{p} d x .
\end{aligned}
$$

Together $\quad \liminf _{n \rightarrow \infty} \int_{\mathbb{R}^{N}}\left|\nabla u_{n}\right|^{p} d x \geq \int_{\mathbb{R}^{N}}|\nabla \bar{u}|^{p} d x$, we get $\lim _{n \rightarrow \infty} \int_{\mathbb{R}^{N}}\left|\nabla u_{n}\right|^{p} d x=\int_{\mathbb{R}^{N}}|\nabla \bar{u}|^{p} d x$. This completes the proof. 


\section{Exponential decay of solutions of Eq. (1.1)}

The main result of this section is the following theorem

Theorem 5.1. Suppose $V$ and $K$ satisfy $\left(\mathbf{A}_{\mathbf{1}}\right)$ and $\left(\mathbf{A}_{\mathbf{2}}\right)$. If $u \in E$ is a weak solution of (1.1), then there exist $C>0$ and $\gamma>0$ such that

$$
|u(x)| \leq C e^{-\gamma|x|^{1-\frac{b}{p}}}, \quad x \in \mathbb{R}^{N} .
$$

To prove this theorem, we need some lemmas.

Lemma 5.2. Suppose $V$ and $K$ satisfy $\left(\mathbf{A}_{\mathbf{1}}\right)$ and $\left(\mathbf{A}_{\mathbf{2}}\right)$. If $u \in E$ is a weak solution of (1.1), then there exist $\alpha>\max \left\{|s|+q|b|(N-p) / p^{2}, p|s| /(q-p)\right\}$, $C>0$, and $R_{0}>0$ such that for $R>R_{0}$,

$$
\int_{|x| \geq R} K|u|^{q} d x \leq C R^{-\alpha} .
$$

Proof. Let $\phi \in C^{\infty}(\mathbb{R})$ be such that

$$
0 \leq \phi \leq 1 ; \phi(r)=0, r \in(-\infty, 0] ; \phi(r)=1, r \in[1,+\infty) ; 0 \leq \phi^{\prime}(r) \leq 2 .
$$

For $R^{\prime}>L$ and $n \in \mathbb{N}$, let $\eta_{n}(x)=\phi\left(|x| / 2^{n} R^{\prime}\right), x \in \mathbb{R}^{N}$. Then supp $\left|\nabla \eta_{n}\right|$, the support of $\left|\nabla \eta_{n}\right|$, is contained in $\left\{x \in \mathbb{R}^{N}\left|2^{n} R^{\prime} \leq\right| x \mid \leq 2^{n+1} R^{\prime}\right\}$. Using $\eta_{n}^{p} u$ as a test function on the Eq. (1.1), we obtain

$$
\int_{\mathbb{R}^{N}}|\nabla u|^{p-2} \nabla u \nabla\left(\eta_{n}^{p} u\right) d x+\int_{\mathbb{R}^{N}} V(x)\left|\eta_{n} u\right|^{p}=\int_{\mathbb{R}^{N}} K(x)|u|^{q-2} u \cdot\left(\eta_{n}^{p} u\right) d x .
$$

As in the proof of (3.16), we have

$$
\begin{aligned}
\int_{\mathbb{R}^{N}}|\nabla u|^{p-2} \nabla u \nabla\left(\eta_{n}^{p} u\right) d x \geq & \frac{1}{4} \int_{\mathbb{R}^{N}}\left|\nabla\left(\eta_{n} u\right)\right|^{p} d x \\
& -\frac{C}{\left(2^{n} R^{\prime}\right)^{p}} \int_{2^{n} R^{\prime} \leq|x| \leq 2^{n+1} R^{\prime}}|u|^{p} d x,
\end{aligned}
$$

where $C>0$ is independent of $n$ and $R^{\prime}$

From $u \in E$, we have $\int_{\mathbb{R}^{N}} K(x)|u|^{q} d x<+\infty$. It follows that there exists $R_{0}>0$ such that, for $R^{\prime}>R_{0}$ and $n \geq 1$,

$$
S^{-1}\left(\int_{|x| \geq 2^{n} R^{\prime}} K(x)|u|^{q} d x\right)^{\frac{q-p}{q}} \leq \frac{1}{8} .
$$

And the similar proof for (3.20) yields

$$
\int_{\mathbb{R}^{N}} K(x)|u|^{q-2} u \cdot\left(\eta_{n}^{p} u\right) d x \leq S^{-1}\left(\int_{|x| \geq 2^{n} R^{\prime}} K(x)|u|^{q}\right)^{\frac{q-p}{q}}\left\|\eta_{n} u\right\|_{E}^{p}
$$

Combining (5.3)-(5.6), we obtain

$$
\begin{aligned}
\int_{|x| \geq 2^{n+1} R^{\prime}} V(x)|u|^{p} d x & \leq \int_{\mathbb{R}^{N}}\left|\nabla\left(\eta_{n} u\right)\right|^{p} d x+\int_{\mathbb{R}^{N}} V(x)\left|\eta_{n} u\right|^{p} d x \\
& \leq \frac{C}{\left(2^{n} R^{\prime}\right)^{p}} \int_{2^{n} R^{\prime} \leq|x| \leq 2^{n+1} R^{\prime}}|u|^{p} d x .
\end{aligned}
$$


From $\left(\mathbf{A}_{\mathbf{2}}\right)$, there exists $C>0$ which is independent of $R^{\prime}$ and $n$, such that for $R^{\prime}>R_{0}$ and $n \geq 1$,

$$
\int_{2^{n} R^{\prime} \leq|x| \leq 2^{n+1} R^{\prime}}|u|^{p} d x \leq C\left(2^{n} R^{\prime}\right)^{b} \int_{2^{n} R^{\prime} \leq|x| \leq 2^{n+1} R^{\prime}} V(x)|u|^{p} d x .
$$

Let $a_{n}=\int_{|x| \geq 2^{n} R^{\prime}} V(x)|u|^{p} d x$. By (5.7) and (5.8), we get that

$$
\frac{a_{n+1}}{a_{n}} \leq \frac{C}{\left(2^{n} R^{\prime}\right)^{p-b}} .
$$

where $C>0$ is independent of $n$ and $R^{\prime}>R_{0}$.

Let $n_{0} \in \mathbb{N}$ be such that

$$
n_{0} \geq \frac{1}{p-b} \max \left\{|s|+q|b|(N-p) / p^{2}, p|s| /(q-p)\right\}+2
$$

and $\alpha=(p-b)\left(n_{0}-1\right)$. Then $\alpha>\max \left\{|s|+q|b|(N-p) / p^{2}, p|s| /(q-p)\right\}$. By (5.9), we obtain

$$
a_{n_{0}} \leq C^{\left(n_{0}-1\right)} 2^{-\frac{n_{0}\left(n_{0}-1\right)}{2}(p-b)}\left(R^{\prime}\right)^{-\alpha} a_{1} \leq C\left(R^{\prime}\right)^{-\alpha} .
$$

where $C>0$ is independent of $n$ and $R^{\prime}>R_{0}$. Combining (5.8), (5.7) with (5.10), we obtain

$$
\int_{\mathbb{R}^{N}}\left|\nabla\left(\eta_{n_{0}} u\right)\right|^{p} d x+\int_{\mathbb{R}^{N}} V(x)\left|\eta_{n_{0}} u\right|^{p} d x \leq \frac{C}{\left(R^{\prime}\right)^{\alpha}}
$$

where $C>0$ is independent of $n$ and $R^{\prime}>R_{0}$. Let $R=2^{n_{0}+1} R^{\prime}$. Then (5.2) follows from (5.11) and (2.1).

Lemma 5.3. Suppose that $V$ and $K$ satisfy $\left(\mathbf{A}_{\mathbf{1}}\right)$ and $\left(\mathbf{A}_{\mathbf{2}}\right)$. If $u \in E$ is a weak solution of (1.1), then there exists $C>0$ such that for $|x|>R_{0}+2$,

$$
|u(x)| \leq C|x|^{-(\alpha-s) / q} .
$$

where $\alpha$ and $R_{0}$ are the constants appearing in Lemma 5.2.

Proof. For $x \in \mathbb{R}^{N}$, let $u^{ \pm}(x)=\max \{ \pm u(x), 0\}$. Then $u^{+}$and $u^{-}$satisfy the inequality

$$
-\Delta_{p} v+V(x) v^{p-1} \leq K(x) v^{q-1} \quad \text { in } \mathbb{R}^{N} .
$$

To prove this lemma, it suffices to prove that $u^{ \pm}$satisfy $(5.12)$.

Take $G(s)=s^{\beta}$ if $s>0$, and zero otherwise, and put

$$
F(s)=\int_{0}^{s} G^{\prime}(t)^{p} d t=\beta^{p} s^{p \beta-p+1} /(p \beta-p+1) .
$$

It is easy to verify that

$$
s^{p-1} F(s) \leq s^{p} G^{\prime}(s)^{p} \leq \beta^{p} G(s)^{p}, \quad \text { if } \beta \geq 1 .
$$

Take $\phi \in C^{\infty}[0,+\infty)$ such that

$$
0 \leq \phi \leq 1 ; \phi(r)=1, r \in[0,1] ; \phi(r)=0, r \in[2,+\infty) ;-2 \leq \phi^{\prime}(r) \leq 0 .
$$


For $\left|x_{0}\right|>R_{0}+2$, let $\eta(x)=\phi\left(\left|x-x_{0}\right|\right), x \in \mathbb{R}^{N}$. Using $\eta^{p} F(v)$ as a test function in the inequality (5.13), we obtain

$$
\begin{aligned}
& \int_{\mathbb{R}^{N}}|\nabla v|^{p} G^{\prime}(v)^{p} \eta^{p} d x+\int_{\mathbb{R}^{N}} V|v|^{p-2} v \cdot \eta^{p} F(v) d x \\
& \quad+p \int_{\mathbb{R}^{N}}|\nabla v|^{p-2} F(v) \eta^{p-1}(\nabla v \nabla \eta) d x \leq \int_{\mathbb{R}^{N}} K v^{q-1} \cdot \eta^{p} F(v) d x .
\end{aligned}
$$

Using the Hölder inequality: For $a, b \geq 0, a b \leq \epsilon a^{\frac{p}{p-1}}+p^{-1}\left(\frac{p}{p-1} \epsilon\right)^{-(p-1)} b^{p}$ and (5.14), we get that

$$
\begin{aligned}
\left.|| \nabla v\right|^{p-2} F(v) \eta^{p-1}(\nabla v \nabla \eta) \mid & \leq \epsilon|\nabla v|^{p} \eta^{p} v^{-1} F(v)+C_{\epsilon}^{\prime} v^{p-1} F(v)|\nabla \eta|^{p} \\
& \leq \epsilon|\nabla v|^{p} \eta^{p} G^{\prime}(v)^{p}+C_{\epsilon}^{\prime} \beta^{p}|\nabla \eta|^{p} G(v)^{p}
\end{aligned}
$$

where $C_{\epsilon}^{\prime}=p^{-1}\left(\frac{p}{p-1} \epsilon\right)^{-(p-1)}$. It follows that

$$
\begin{aligned}
& \left.\int_{\mathbb{R}^{N}}|| \nabla v\right|^{p-2} F(v) \eta^{p-1}(\nabla v \nabla \eta) \mid d x \\
& \leq \epsilon \int_{\mathbb{R}^{N}}|\eta \nabla G(v)|^{p} d x+C_{\epsilon}^{\prime} \beta^{p} \int_{\mathbb{R}^{N}}|\nabla \eta|^{p} G(v)^{p} d x \\
& \leq \epsilon \int_{\mathbb{R}^{N}}|\eta \nabla G(v)|^{p} d x+C_{\epsilon} \beta^{p}\left(\int_{B\left(x_{0}, 2\right)}|G(v)|^{q} d x\right)^{\frac{p}{q}} .
\end{aligned}
$$

where $C_{\epsilon}>0$ depends only on $N, \epsilon$ and $p$. Using a similar proof as for (3.16), we have

$$
\begin{aligned}
\int_{\mathbb{R}^{N}}|\nabla v|^{p} G^{\prime}(v)^{p} \eta^{p} d x & \geq \frac{1}{2} \int_{\mathbb{R}^{N}}|\nabla(\eta G(v))|^{p} d x-C \int_{\mathbb{R}^{N}}|G(v)|^{p}|\nabla \eta|^{p} d x \\
& \geq \frac{1}{2} \int_{\mathbb{R}^{N}}|\nabla(\eta G(v))|^{p} d x-C\left(\int_{B\left(x_{0}, 2\right)}|G(v)|^{q} d x\right)^{\frac{p}{q}}
\end{aligned}
$$

where $C>0$ depends only on $p$ and $N$. Moreover, by (5.14), we get that

$$
\begin{aligned}
& \int_{\mathbb{R}^{N}} K v^{q-1} \cdot \eta^{p} F(v) d x=\int_{\mathbb{R}^{N}} K v^{q-p} v^{p-1} \cdot \eta^{p} F(v) d x \\
& \leq \beta^{p} \int_{\text {supp } \eta} K|v|^{q-p}(\eta G(v))^{p} d x \\
& \leq \beta^{p}\left(\int_{\text {supp } \eta} K^{\frac{q}{q-p}}|v|^{q} d x\right)^{\frac{q-p}{q}}\left(\int_{B\left(x_{0}, 2\right)}|G(v)|^{q} d x\right)^{\frac{p}{q}} .
\end{aligned}
$$

Note that $\left|x_{0}\right|-2>R_{0}$ and supp $\eta$ is contained in $\left\{x|| x|>| x_{0} \mid-2\right\}$. From (5.2), we have that (choose $R=\left|x_{0}\right|-2$ )

$$
\int_{\text {supp } \eta} K|v|^{q} d x \leq C \int_{|x|>\left|x_{0}\right|-2} K|v|^{q} d x \leq C\left|x_{0}\right|^{-\alpha} .
$$


By $\left(\mathbf{A}_{\mathbf{2}}\right)$, there exists $C>0$ independent of $x_{0}$, such that,

$$
K(x) \leq C\left|x_{0}\right|^{-s}, \forall x \in B\left(x_{0}, 2\right) .
$$

Together with (5.19) and the fact that $-\frac{p}{q} s-\frac{q-p}{q} \alpha<0$, this yields

$$
\left(\int_{\text {supp } \eta} K^{\frac{q}{q-p}}|v|^{q} d x\right)^{\frac{q-p}{q}} \leq C\left|x_{0}\right|^{-\frac{p}{q} s-\frac{q-p}{q} \alpha} \leq C
$$

where $C>0$ is independent of $x_{0}$. Then, by (5.18), we obtain

$$
\int_{\mathbb{R}^{N}} K v^{q-1} \cdot \eta^{p} F(v) d x \leq C \beta^{p}\left(\int_{B\left(x_{0}, 2\right)}|G(v)|^{q} d x\right)^{\frac{p}{q}} .
$$

Choosing $\epsilon=1 / 4 p$ in (5.16) and combining (5.15), (5.16), (5.17) with (5.22), we obtain

$$
\int_{\mathbb{R}^{N}}|\nabla(\eta G(v))|^{p} d x \leq C\left(1+\beta^{p}\right)\left(\int_{B\left(x_{0}, 2\right)}|G(v)|^{q} d x\right)^{\frac{p}{q}} .
$$

Then, using the Sobolev inequality, we get

$$
\left(\int_{B\left(x_{0}, 1\right)}|G(v)|^{p^{*}} d x\right)^{\frac{p}{p^{*}}} \leq C\left(1+\beta^{p}\right)\left(\int_{B\left(x_{0}, 2\right)}|G(v)|^{q} d x\right)^{\frac{p}{q}}
$$

where $C>0$ is independent of $x_{0}$ and $\beta$. From this, the standard Moser's iteration (see [14, Theorem 8.18]) shows that for $x \in B\left(x_{0}, 1 / 2\right)$,

$$
v(x) \leq C\left(\int_{B\left(x_{0}, 2\right)}|v|^{q} d x\right)^{\frac{1}{q}}
$$

where $C>0$ is independent of $x_{0}$. By $\left(\mathbf{A}_{2}\right), K(x) \geq C\left|x_{0}\right|^{-s}, \forall x \in B\left(x_{0}, 2\right)$. Then by (5.19) and (5.20), we deduce that

$$
\left(\int_{B\left(x_{0}, 2\right)}|v|^{q} d x\right)^{\frac{1}{q}} \leq C\left|x_{0}\right|^{\frac{-\alpha+s}{q}}
$$

where $C>0$ is independent of $x_{0}$. The result of this lemma follows form the above two inequalities.

Lemma 5.4. Suppose $V$ satisfies $\left(\mathbf{A}_{\mathbf{1}}\right)$ and $\left(\mathbf{A}_{\mathbf{2}}\right)$. There exists $R_{*}>L$ such that, if $u \in E$ is a nonnegative and locally bounded function satisfying the inequality:

$$
-\Delta_{p} u+\frac{1}{2} V(x) u^{p-1} \leq 0, \quad|x|>R_{*}
$$

then there exist $\gamma>0$ and $C>0$ such that

$$
u(x) \leq C e^{-\gamma|x|^{1-\frac{b}{p}}}, \quad|x|>R_{*} .
$$


Proof. For $\epsilon>0$ and $r=|x|$, let $w_{\epsilon}(r)=e^{-\epsilon r^{1-\frac{b}{p}}}$. Direct computation shows that

$$
\begin{aligned}
\Delta_{p} w_{\epsilon}(|x|) & =\left|w_{\epsilon}^{\prime}(r)\right|^{p-2}\left((p-1) w_{\epsilon}^{\prime \prime}(r)+\frac{N-1}{r} w_{\epsilon}^{\prime}(r)\right) \\
& =\epsilon^{p} B_{1} r^{-b} e^{-(p-1) r^{1-\frac{b}{p}}}+\epsilon^{p-1} B_{2} r^{-b-\left(1-\frac{b}{p}\right)} e^{-(p-1) r^{1-\frac{b}{p}}}
\end{aligned}
$$

where

$$
B_{1}=(p-1)\left(1-\frac{b}{p}\right)^{p}, B_{2}=\left(1-\frac{b}{p}\right)^{p-1}\left(b-\frac{b}{p}-N+1\right) .
$$

Then by $\left(\mathbf{A}_{\mathbf{2}}\right)$, we can choose $R_{*}>L$ and a sufficiently small $\epsilon_{0}>0$ such that for $|x| \geq R_{*}$,

$$
-\Delta_{p} w_{\epsilon_{0}}+\frac{1}{2} V(x) w_{\epsilon_{0}}^{p-1} \geq 0
$$

Since $u$ is a locally bounded function, we can choose $\lambda>0$ such that

$$
\bar{u}:=\lambda w_{\epsilon_{0}}>u \quad \text { for }|x|=R_{*} .
$$

It follows that $(\bar{u}-u)^{-} \in E$. By (5.23) and (5.25), we have

$$
-\Delta_{p} \bar{u}+\Delta_{p} u+\frac{1}{2} V(\bar{u}-u) \geq 0 .
$$

Multiplying both sides of this inequality by $(\bar{u}-u)^{-}$and integrating in $\left\{x|| x \mid>R_{*}\right\}$, we obtain

$$
\begin{aligned}
& \int_{\Omega}\left(|\nabla \bar{u}|^{p-2} \nabla \bar{u}-|\nabla u|^{p-2} \nabla u\right)(\nabla \bar{u}-\nabla u) d x \\
& \quad+\frac{1}{2} \int_{\Omega} V(x)\left(|\bar{u}|^{p-2} \bar{u}-|u|^{p-2} u\right)(\bar{u}-u) d x \leq 0,
\end{aligned}
$$

where $\Omega=\left\{x|\bar{u}(x)-u(x)<0| x \mid,>R_{*}\right\}$. From [19], there exists $c>0$ such that for any $x, y \in \mathbb{R}^{m}(m \geq 1)$,

$$
\left(|x|^{p-2} x-|y|^{p-2} y\right) \cdot(x-y) \geq \begin{cases}c|x-y|^{p}, & p \geq 2 \\ c \frac{|x-y|^{2}}{(|x|+|y|)^{p-2}}, & 1<p<2 .\end{cases}
$$

By this inequality and (5.26), we deduce that $\Omega$ has zero measure. The result of this lemma follows.

Proof of Theorem 5.1. Since $V$ and $K$ are locally bounded functions and $q<p^{*}$, by the classical regularity theory of quasilinear elliptic equations (see, for example, [27]), we have $u \in L_{l o c}^{\infty}\left(\mathbb{R}^{N}\right)$.

By assumption, $u$ satisfies

$$
-\Delta_{p} u+\left(V-K|u|^{q-p}\right)|u|^{p-2} u=0 \quad \text { in } \mathbb{R}^{N} .
$$

By the definition of $\alpha$ (see Lemma 5.2), we have

$$
\frac{\alpha-s}{q}(q-p)+s>b .
$$


Together with Lemma 5.3, this implies that there exists $R_{* *}>R_{*}$ such that $V-K|u|^{q-p}>V / 2$ if $|x| \geq R_{* *}$. It follows that $u^{+}$satisfies

$$
-\Delta_{p} u^{+}+\frac{1}{2} V(x)\left(u^{+}\right)^{p-1} \leq 0 \text { if }|x| \geq R_{* *} .
$$

Then, Lemma 5.4 implies that there exist $C>0$ and $\gamma>0$ such that $u^{+}(x) \leq$ $C e^{-\gamma|x|^{1-\frac{b}{p}}}$ if $|x| \geq R_{* *}$. Similarly, we can prove that $u^{-}(x) \leq C e^{-\gamma|x|^{1-\frac{b}{p}}}$ if $|x| \geq R_{* *}$. The result of this theorem follows from these two inequalities and fact that $u \in L_{\text {loc }}^{\infty}\left(\mathbb{R}^{N}\right)$.

\section{Acknowledgements}

The authors would like to thank the anonymous referees for their useful comments and suggestions on the manuscript. The first author was supported by Science Foundation of Huaqiao University and Promotion Program for Young and Middle-aged Teacher in Science and Technology Research of Huaqiao University (ZQN-PY119). The second author is supported by NSFC-11271201.

\section{References}

[1] Adams, R., Fournier, J.F.: Sobolev Spaces, 2nd ed. In: Pure and Applied Mathematics (Amsterdam), vol. 140. Elsevier/Academic Press, Amsterdam (2003)

[2] Alves, C.O.: Existence of multi-bump solutions for a class of quasilinear problems. Adv Nonlinear Stud. 6, 491-509 (2006)

[3] Alves, C.O., Souto, M.S.: Existence of solutions for a class of nonlinear Schrödinger equations with potential vanishing at infinity. J. Differ. Equ. 254, 1977-1991 (2013)

[4] Ambrosetti, A., Wang, Z.-Q.: Nonlinear Schrödinger equations with vanishing and decaying potentials. Differ. Integral Equ. 18, 1321-1332 (2005)

[5] Ambrosetti, A., Felli, V., Malchiodi, A.: Ground states of nonlinear Schrödinger equations with potentials vanishing at infinity. J Eur. Math. Soc. 7, 117$144(2005)$

[6] Astarita, G., Marrucci, G.: Principles of Non-Newtonian Fluid Mechanics. McGraw-Hill, New York (1974)

[7] Bartsch, T., Wang, Z.-Q.: Existence and multiplicity results for some superlinear elliptic problems on $\mathbb{R}^{N}$. Comm Partial Differ. Equ. 20, 1725-1741 (1995)

[8] Bartsch, T., Pankov, A., Wang, Z.-Q.: Nonlinear Schrödinger equations with steep potential well. Commun. Contemp. Math. 3, 549-569 (2001)

[9] Bonheure, D., Van Schaftingen, J.: Groundstates for the nonlinear Schrödinger equation with potential vanishing at infinity. Ann. Mat. Pura Appl. 189(4), 273$301(2010)$ 
[10] Byeon, J., Wang, Z.-Q.: Standing waves with a critical frequency for nonlinear Schrödinger equations. Arch. Ration. Mech. Anal. 165, 295-316 (2002)

[11] Chen, S.: Existence of positive solutions for a critical nonlinear Schrödinger equation with vanishing or coercive potentials. Bound. Value Probl. 2013, 201 2013

[12] Costa, D.G.: On a class of elliptic systems in $\mathbb{R}^{N}$. Electron. J. Differ. Equ. 7, 114 (1994)

[13] Felmer, P., Mayorga-Zambrano, J.: Multiplicity and concentration for the nonlinear Schrödinger equation with critical frequency. Nonlinear Anal. 66, 151$169(2007)$

[14] Gilbarg, D., Trudinger, N.: Elliptic partial differential equations of second order. Reprint of the 1998 edition. In: Classics in Mathematics. Springer, Berlin (2001)

[15] Lyberopoulos, A.N.: Quasilinear scalar field equations with competing potentials. J Differ. Equ. 251, 3625-3657 (2011)

[16] Lyberopoulos, A.N.: Bound states for a class of quasilinear scalar field equations with potentials vanishing at infinity. Acta Math. Sci. Ser. B Engl. Ed. 32, 197$208(2012)$

[17] Opic, B., Kufner, A.: Hardy-type inequalities, Pitman Res. Notes in Mathematical Series, vol. 219. Longman Scientific Technical, Harlow (1990)

[18] Rabinowitz, P.: Minimax methods in critical point theory with applications to differential equations. In: CBMS Regional Conference Series in Mathematics, vol. 65. American Mathematical Society, Providence (1986)

[19] Simon, J.: Régularité de la Solution d'une Équation non Linéaire Dans $\mathbb{R}^{N}$ In: Lectures Notes in Mathematics, vol. 665, pp. 205-227. Springer, Berln (1978)

[20] Sintzoff, P.: Symmetry of solutions of a semilinear elliptic equation with unbounded coefficients. Differ. Integral Equ. 16, 769-786 (2003)

[21] Sintzoff, P., Willem, M.: A semilinear elliptic equation on $\mathbb{R}^{N}$ with unbounded coefficients in: variational and toplogical methods in the study of nonlinear phenomena. In: Progress in Nonlinear Differential Equations and their Applications, vol. 49. Birkhäuser Boston, Inc., Boston (2002)

[22] Sirakov, B.: Existence and multiplicity of solutions of semi-linear elliptic equations in $\mathbb{R}^{N}$. Calc. Var. Partial Differ. Equ. 11, 119-142 (2000)

[23] Souplet, P., Zhang, Q.S.: Stability for semilinear parabolic equations with decaying potentials in $\mathbb{R}^{n}$ and dynamical approach to the existence of ground states. Ann. Inst. H. Poincaré Anal. Non Linéaire. 19, 683-703 (2002)

[24] Su, J., Wang, Z.-Q.: Sobolev type embedding and quasilinear elliptic equations with radial potentials. J. Differ. Equ. 250, 223-242 (2011) 
[25] Su, J., Wang, Z.-Q., Willem, M.: Nonlinear Schrödinger equations with unbounded and decaying radial potentials. Commun. Contemp. Math. 9, 571$583(2007)$

[26] Su, J., Wang, Z.-Q., Willem, M.: Weighted Sobolev embedding with unbounded and decaying radial potentials. J. Differ. Equ. 238, 201-219 (2007)

[27] Tolksdorf, P.: Regularity for a more general class of quasilinear elliptic equations. J. Differ. Equ. 51, 126-150 (1984)

[28] Willem, M.: Minimax theorems. In: Progress in Nonlinear Differential Equations and their Applications, vol. 24. Birkhäuser Boston, Inc., Boston, MA (1996)

[29] Zhang, G.: Weighted Sobolev spaces and ground state solutions for quasilinear elliptic problems with unbounded and decaying potentials, Bound. Value Probl. 2013, 189 (2013)

[30] Zhang, Q.S.: Positive solutions to $\Delta u-V u+W u^{p}=0$ and its parabolic counterpart in noncompact manifolds. Pacific J. Math. 213, 163-200 (2004)

Shaowei Chen

School of Mathematical Sciences

Huaqiao University

Quanzhou 362021

People's Republic of China

e-mail: swchen6@163.com

Zhi-Qiang Wang

Center for Applied Mathematics

Tianjin University

Tianjin 300072

People's Republic of China

e-mail: zhi-qiang.wang@usu.edu

Zhi-Qiang Wang

Department of Mathematics and Statistics

Utah State University

Logan

UT 84322

USA

Received: 1 July 2014.

Accepted: 12 November 2014. 